\title{
REPERCUSIONES DE UN FENOMENO EXTRAORDINARIO EN LA RAMBLA DE CHIVA
}

\author{
F. Segura* \\ E. Sanjaume* \\ M.J. Meyer*
}

\begin{abstract}
RESUMEN
El presente artículo intenta demostrar que las avenidas sólo se convierten en desastres cuando coadyuvan acciones antrópicas. Para confirmar esta hipótesis se han estudiado las causas y las consecuencias de una inundación producida en la Rambla de Chiva. Una parte del artículo se dedica a analizar las influencias que dicho fenómeno ha tenido sobre los sedimentos de la propia rambla. Este estudio permite concluir que las inundaciones son un fenómeno normal en el funcionamiento de los ríos. El resto se dedica a analizar la incidencia de la actuación humana sobre la rambla y las repercusiones que de ella se derivan.
\end{abstract}

\section{SUMMARY}

In this paper we have tried to show that floods only become catastrophic when man acts on them. In order to confirm such hypotesis causes and consequences of a flood occurred at the Rambla de Chiva have been analysed. The first part of the article studies the consequences of such phenomenon on the rambla by means of the sedimentology. From this study it can be concluded that floods are a normal phenomenon for river action. The second part is centered in human intervention on the Rambla, which is shown to have had catastrophic effects on it.

* Departamento de Geografía. Valencia 
La violenta crecida de la Rambla de Chiva el 9 de noviembre de 1983, corresponde a un tipo de sucesos bastante frecuentes en el ámbito mediterráneo. Buena prueba de ello son las inundaciones catastróficas acaecidas en Cataluña, Murcia y País Valenciano en octubre-noviembre de 1982. En la ribera del Xúquer ha quedado constancia documental de más de 80 desbordamientos desde 1270 (MATEU, 1983, p. 292). En las últimas décadas la Rambla de Chiva ha producido tres avenidas de cierta importancia.

La del 28 de septiembre de 1949 causó tres víctimas mortales y se produjo a mediodía. Los materiales de derribo y deshecho, que después de la Guerra Civil habían sido vertidos a la rambla, obstruyeron el cauce agravando la crecida puesto que dificultaban la libre circulación del agua. Una nueva avenida acaeció el 14 de octubre de 1957 coincidiendo con la gran riada del Turia. Al contrario de lo que ocurrió en Valencia y alrededores. en esta ocasión la rambla no causó desgracias personales. ya que toda el agua se canalizó por el cauce. La última avenida se produjo la noche del 9 de noviembre de 1983, como consecuencia de las fuertes precipitaciones caídas en pocas horas especialmente en la cuenca del Barranco de Cañadillas, en la zona denominada la Serratilla.

Al igual que ocurrió con la riada del Xúquer - cuya cuenca limita con la rambla - a las causas puramente climáticas habría que añadir la escasa infiltración debida a las precipitaciones de días anteriores, que favoreció la circulación superficial, y las modificaciones antrópicas del cauce que impidieron la libre circulación del agua.

\section{Aspectos generales de la cuenca}

En la Rambla de Chiva se pueden distinguir diversos elementos geomorfológicos. Por un lado podemos diferenciar el cauce principal y por otro sus afluentes. La rambla nace de la confluencia del Barranco del Gallo-que se inicia a $840 \mathrm{~m}$. de altitud, en la Sierra de la Cabrera-y del Barranco Grande que comienza a $900 \mathrm{~m}$. en la Sierra de los Bosques, ambas de orientación ibérica (fig. 1). Tiene una longitud total de $60 \mathrm{Km}$. y una pendiente media del $1,33 \%$.

En la parte alta de la cuenca los barrancos van encajados en materiales calizos y margosos del Cretácico. A la salida al llano el cauce del Barranco del Gallo llega a desaparecer entre Buñol y Chiva, obstruido por potentes conos formados en sus afluentes que descienden de las citadas sierras. Reaparece en las inmediaciones de Chiva, donde a su paso por esta población se encaja de forma espectacular en las areniscas y conglomerados pliocenos. Aguas abajo se une al Barranco Grande y se encaja en margas y arcillas miocenas. La rambla desaparece de nuevo en el término municipal de Manises, sin llegar a confluir con el Barranco del Gállego. Su cauce se desvanece en una superficie cuaternaria antigua, aunque el agua sigue la línea de máxima 
pendiente hasta confluir con el Barranc de Torrent, que se encaja fuertemente en materiales cuaternarios. En cualquier caso hay que hacer notar que parece que la Rambla de Chiva y sus afluentes han desmantelado la superficie pliocena haciendo aflorar los materiales miocenos subyacentes.

Por lo que respecta a sus afluentes, podemos distinguir dos grandes conjuntos. Por una parte, los afluentes que drenan las sierras de cabecera cuyos cauces están perfectamente encajados en los materiales predominantemente calizos del Cretácico. Por otro, los afluentes que circulan por los materiales miocenos - arcillas y margas - y pliocenos (conglomerados y areniscas). En el último caso el agua se encauza por barrancos de fondo plano, lo que hace disminuir la densidad de drenaje que sólo computa los cauces bien definidos.

El excepcional desarrollo de dichos barrancos en esta área es un elemento muy a tener en cuenta, sobre todo en los momentos de crecida. En estos casos excepcionales la importante anchura y rugosidad de estos cauces —dado que la mayoría están abancalados y cultivados- pueden agravar tales sucesos. De hecho, las avenidas que inundaron Chiva en 1949 y 1983 se canalizaron por el Barranco de las Cañadillas que es de fondo plano.

Desde el punto de vista de la morfometría, la longitud total de los cauces es de $415 \mathrm{~km}$., de los cuales 60 corresponden al canal principal. Relacionados con los $462 \mathrm{Km}^{2}$ del área de la cuenca se obtiene una densidad de drenaje de $0,89 \mathrm{Km} / \mathrm{Km}^{2}$. Como puede observarse en la fig. 1, la parte alta de la cuenca tiene una densidad superior a la del resto de la rambla. Para poder cuantificar estas diferencias se han hallado las densidades de drenaje de cada zona. En la parte alta es de $1,77 \mathrm{Km} / \mathrm{Km}^{2}$, en tanto que en la parte baja es sólo de $0,65 \mathrm{Km} / \mathrm{Km}^{2}$. Estas diferencias pueden deberse al cambio de litología señalado anteriormente.

Por otra parte, se ha establecido la jerarquía de los cauces siguiendo el método propuesto por STRAHLER. A partir de un número de orden se ha hallado la relación de bifurcación y a partir de la extensión de los cauces, la relación de longitud. Los resultados obtenidos, pese a la pequeñez del área, se expresan en el siguiente cuadro:

\begin{tabular}{|c|c|c|c|c|c|}
\hline $\begin{array}{c}\text { N." } \\
\text { orden }\end{array}$ & $\begin{array}{c}\text { N." } \\
\text { cauces }\end{array}$ & $\begin{array}{c}\text { Relación } \\
\text { bifurcación }\end{array}$ & Longitud & $\begin{array}{c}\text { Longitud } \\
\text { media }\end{array}$ & $\begin{array}{c}\text { Relación } \\
\text { longitud }\end{array}$ \\
\hline 1 & 144 & 3.78 & 217 & 1,51 & 1,39 \\
2 & 38 & 6,33 & 80 & 2,11 & 4,81 \\
3 & 6 & 3,00 & 61 & 10,16 & 0,78 \\
4 & 2 & 2,00 & 16 & 8,00 & 5,00 \\
5 & 1 & & 40 & 40,00 & \\
\hline
\end{tabular}




\section{F. SEGURA, E. SANJAUME, M.J. MEYER}

Como puede comprobarse, se dan una serie de anomalías en algunas relaciones. La de bifurcación es más alta de lo normal cuando se compara el orden 2 y el 3. Por otro lado, la relación de longitud entre los órdenes 3 y 4 es anormalmente inferior a uno. De esto se deduce que estas alteraciones están determinadas por el excesivo desarrollo en longitud y la ausencia de afluentes en los cauces de orden 3. Las relaciones de área -aunque no se han obtenido para el presente trabajo- dadas las características de los anteriores parámetros, es presumible que también fueran anómalas en los cauces del citado orden.

Por último, otro elemento que caracteriza esta red fluvial es la disposición ortogonal y disimétrica de los afluentes de cabecera. Ello puede tener una considerable importancia en los momentos de precipitaciones extraordinarias, dado que drenan una reducida área montañosa y ello puede hacer coincidir sus picos de crecida, desbordando la capacidad del cauce.

\section{Condicionantes meteorológicos e hidrológicos}

La cuenca de esta rambla goza de un clima típicamente mediterráneo, caracterizado por altas temperaturas y precipitaciones escasas. La evapotranspiración es también elevada. Por lo tanto el agua que queda para circular en superficie o subterráneamente es muy escasa, como lo demuestra el balance hidrológico obtenido.

Las precipitaciones anuales de los observatorios de la cuenca oscilan entre los $505 \mathrm{~mm}$. de Buñol y los $418 \mathrm{~mm}$. de Manises, mientras que la evapotranspiración - que aumenta con la disponibilidad de agua - varía entre $485 \mathrm{~mm}$. y $418 \mathrm{~mm}$. respectivamente. En la mayoría de los observatorios, sin embargo, ambos datos coinciden. Sólo en los de Buñol, Chiva y Torís la evapotranspiración es inferior a la precipitación.

Para obtener el balance hidrológico de la cuenca se han determinado los valores medios de estos dos parámetros. Para ello se han utilizado los polígonos de THIESSEN, a fin de ponderar los datos teóricos con la superficie representativa de cada observatorio. Las cifras obtenidas son: 447,45 mm. de precipitación y $437,28 \mathrm{~mm}$. de evapotranspiración. Aplicando estos valores a la superficie total de la cuenca, la precipitación anual es de $206,7 \mathrm{Hm}^{3}$ y la evapotranspiración es de $202,02 \mathrm{Hm}^{3}$, por lo que sólo quedan $4,69 \mathrm{Hm}^{3}$ libres para circular.

Distinta es la situación en los momentos en que se producen lluvias torrenciales. PEREZ CUEVA y ARMENGOT (1983, p. 56) han encontrado 21 situaciones de precipitación torrencial en la cuenca baja del Xúquer entre 1949-81, lo cual da una clara idea de la frecuencia con que se producen estos fenómenos extraordinarios en el área. 
Sin embargo, el umbral necesario para que "funcionen" las ramblas es todavía una incógnita. MATEU (1982, p. 136) ha aventurado unos valores mínimos para las ramblas del norte del País Valenciano. Según este autor, una precipitación de $60 \mathrm{~mm}$. concentrada en cinco o seis horas es suficiente para que circule una rambla. Igual sucede con $100 \mathrm{~mm}$. caídos en 24 horas. En cambio, cuando esta última cantidad se concentra en cinco o seis horas se produce una "avenida extraordinaria".

Los datos de precipitación correspondientes a las "avenidas extraordinarias" de la Rambla de Chiva ocurridas en 1949, 1957 y 1983, parecen confirmar dichas aseveraciones. El 28 de septiembre de 1949 se midieron en la población de Chiva $163 \mathrm{~mm}$., lo que supone un $37 \%$ de la precipitación media anual. En esta área cayeron pues $24,77 \mathrm{Hm}^{3}$ en un solo día. Sin embargo, estas lluvias no afectaron a la parte baja de la cuenca y las aguas sólo inundaron la población de Chiva. En 1957, en cambio, las precipitaciones cayeron únicamente en la parte baja de la cuenca. Así, Alaquàs recibió $125,5 \mathrm{~mm}$. el día 15 de octubre, mientras que Buñol registró $50 \mathrm{~mm}$. de máxima el día 14. En esta ocasión las aguas de la crecida se canalizaron perfectamente por el cauce.

En noviembre de 1983 las lluvias cayeron preferentemente sobre Chiva y superaron - según estimaciones de la Confederación Hidrográfica del Júcar - los $100 \mathrm{~mm}$, pero concentrados en sólo un cuarto de hora. Las inundaciones afectaron esta vez a toda la cuenca. De todo ello se deduce que las avenidas más peligrosas se producen cuando el agua cae en la cuenca alta. Además, la concentración horaria es también un agravante puesto que el agua satura rápidamente el suelo facilitando la escorrentía superficial.

\section{Efectos de la crecida}

Las precipitaciones estudiadas anteriormente afectaron a una amplia red de barrancos — situada entre la cuenta baja del Xúquer y la del Turiaque vierten en su mayoría directamente a la Albufera de Valencia, y entre los cuales el más importante es la Rambla de Chiva. Las consecuencias de la crecida se dejaron notar especialmente en la cabecera y en la parte baja. En la primera, los mayores desperfectos fueron provocados por las aguas del Barranco de Cañadillas, afluente de la Rambla de Chiva. En la parte baja la inundación se originó a partir de la confluencia del Barranco de Gállego con la Rambla de Chiva (en su tramo denominado Barranco de Poyo).

En la población de Chiva las aguas del Barranco de Cañadillas - de fondo plano- penetraron, con una altura de más de $1,7 \mathrm{~m}$., en el interior de una fábrica textil, situada en la zona industrial de la carretera de Madrid. Hay que hacer constar que dicho establecimiento se encuentra ubicado en el cono del citado barranco obturando su desagüe natural. La antigua carre- 
tera nacional III, la actual variante, la vía del ferrocarril y los edificios existentes en este sector impidieron la evacuación de las aguas hacia la Rambla de Chiva. Por este motivo, todas las calles de la parte alta de la población - de fuerte pendiente- se convirtieron en auténticos barrancos que bajaban hacia el cauce de la rambla que atraviesa esta población. Las aguas entraron en unas 400 viviendas con una altura entre 0,30 y $0,50 \mathrm{~m}$., en general con daños poco importantes, a excepción de algunos comercios. La fuerte pendiente y la consiguiente fuerza de las aguas, determinaron el arrastre y el destrozo de más de cien vehículos. Además, el colector general de la población -que discurre por la parte central del barranco- quedó totalmente inutilizado.

En su tramo inferior afectó a los términos de Quart de Poblet. Xirivclla, Aldaia y Riba-roja. En este caso, los daños afectaron a la agricultura, a algunas factorías del polígono industrial ubicado en las inmediaciones de la carretera nacional III Valencia-Madrid, y a algunas viviendas del barrio del Mas de l'Olivereta - término de Riba-roja - con una altura, en algunos casos, de 1,5 m. La crecida, en este punto, se vio favorecida por la ausencia de un cauce definido, que canalizara de manera adecuada las aguas de la Rambla de Chiva y del Barranco de Poyo, hacia su tramo final del Barranc de Torrent.

\section{Repercusiones de los sedimentos del cauce}

Para averiguar los efectos que la crecida ha tenido en los sedimentos del cauce se ha recurrido a un estudio granulométrico de los mismos. Para ello se ha muestreado una sección transversal del cauce, en uno de los tramos que permanecen sin alteración antrópica. La localización de las muestras se puede observar en la fig. 2.

La muestra 1 se tomó en el extremo distal de la barra, que presentaba una longitud de casi $25 \mathrm{~m}$. y una anchura máxima de $15 \mathrm{~m}$. Tiene un calibre medio de $-5,44 \varphi$, lo que indica un extraordinario predominio de la fracción gruesa. Su histograma de frecuencias es bimodal (fig. 3). Es un sedimento heterométrico, aunque falta casi por completo la fracción arena. Su clasificación es moderada, si bien es la muestra mejor seleccionada de todo este tramo del cauce. La curva acumulada se dibuja netamente parabólica (fig. 4) lo que denota una acumulación forzada. En este caso, además del descenso de la velocidad de la corriente, la existencia de unos árboles en el cauce —que actúan de obstáculo- ha favorecido la deposición de parte de la carga de fondo. Su índice de asimetría $(0,46)$ corrobora el predominio de la decantación sobre el lavado. En esta muestra la principal modalidad de transporte es el rodamiento con el $60 \%$ del total, estableciéndose el punto de ruptura en $-5,5 \varphi$. 


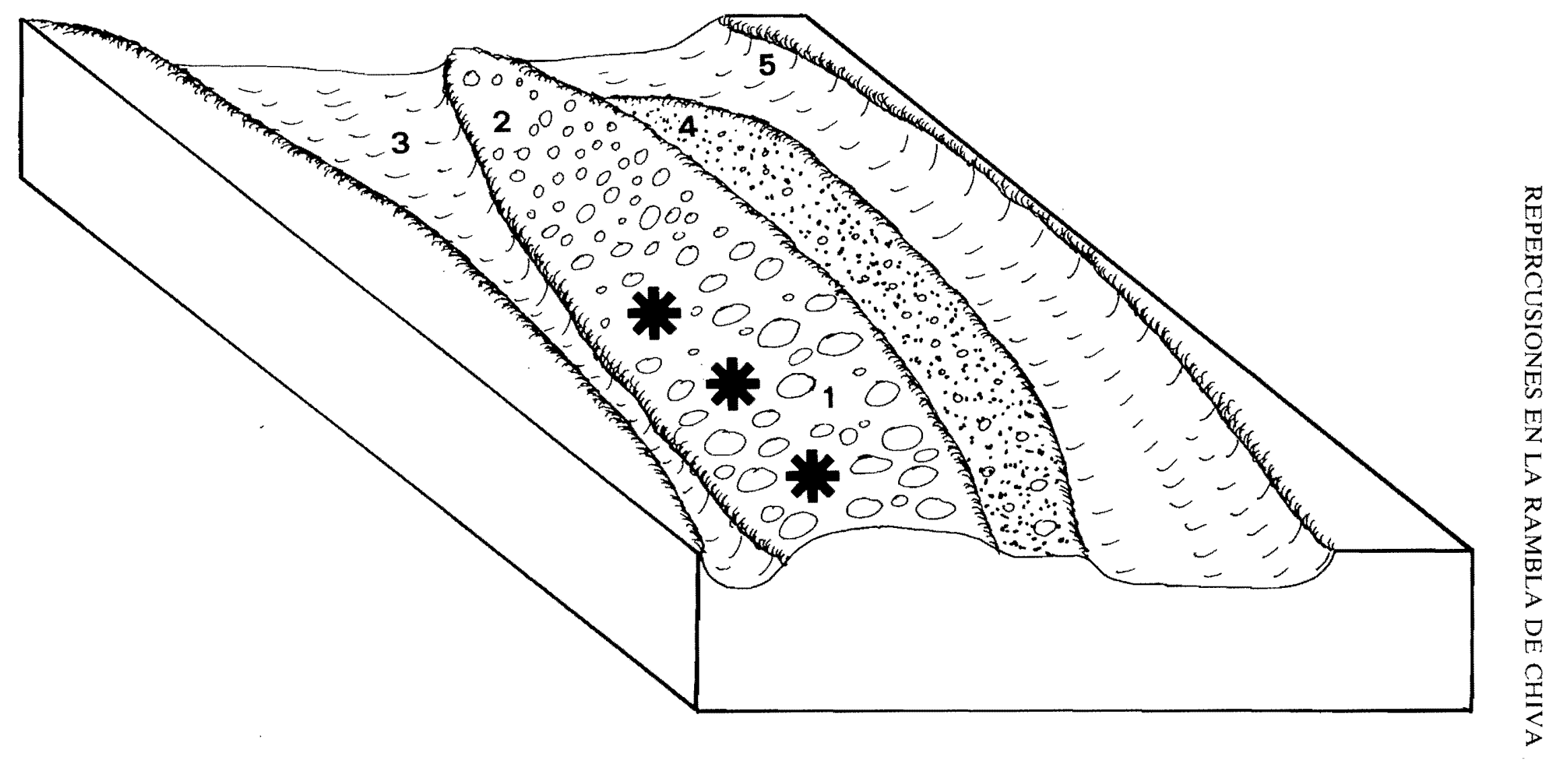

Figura 2

Bloque diagrama con la localización de las muestras. Los asteriscos corresponden a la ubicación de los arboles. 

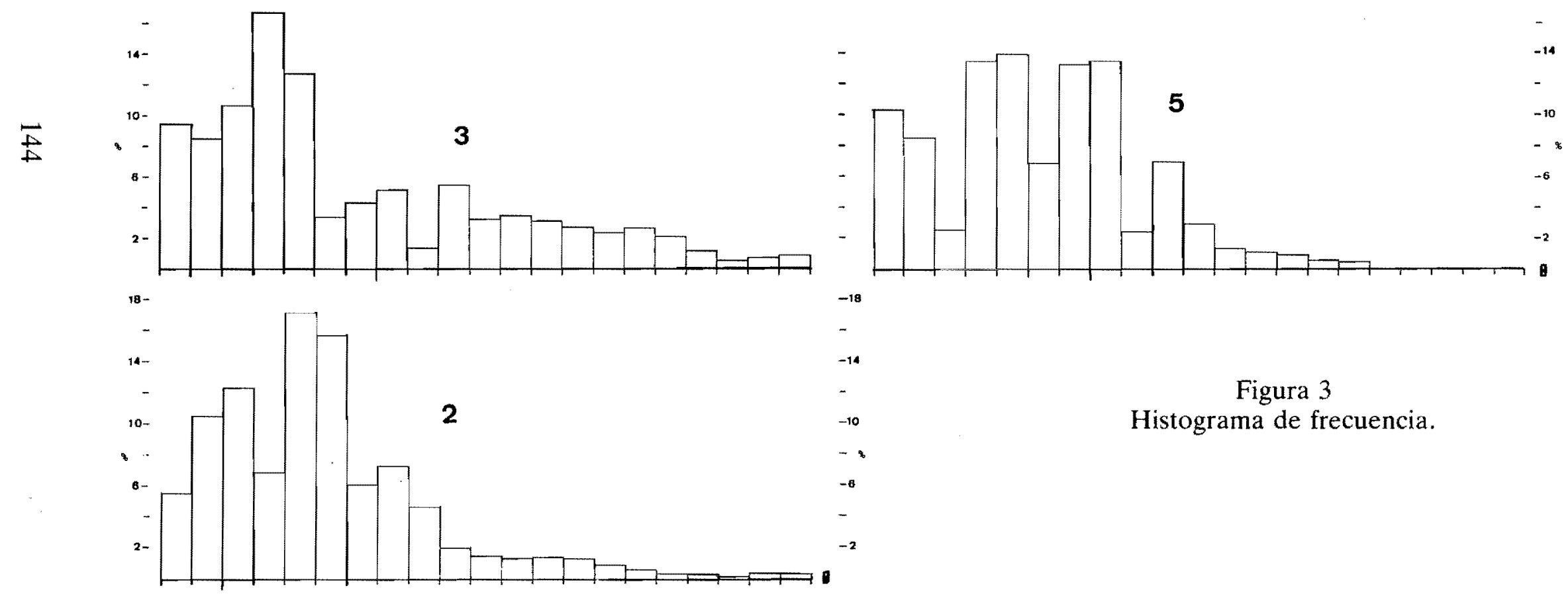

Figura 3
Histograma de frecuencia.
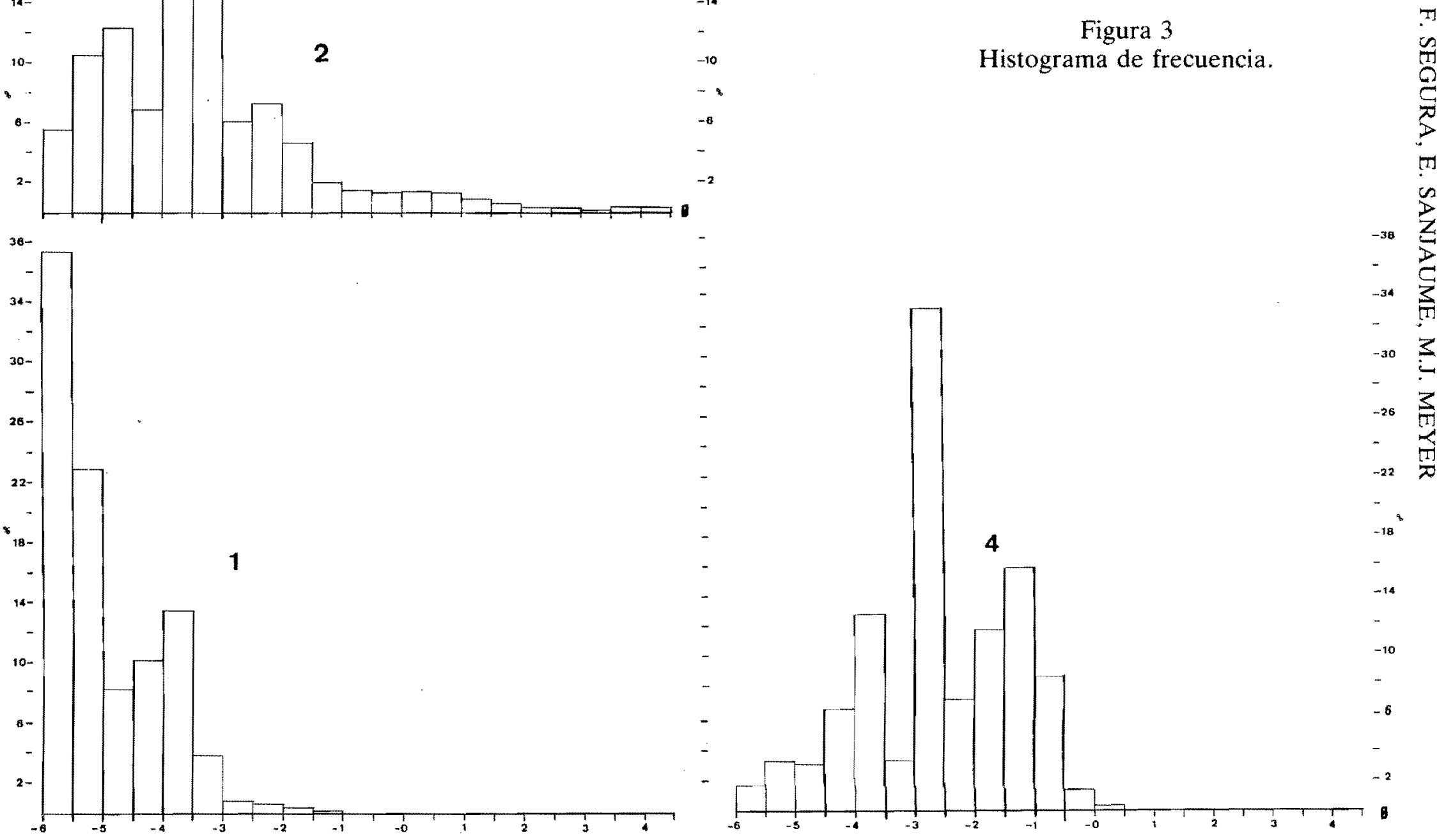
REPERCUSIONES EN LA RAMBLA DE CHIVA

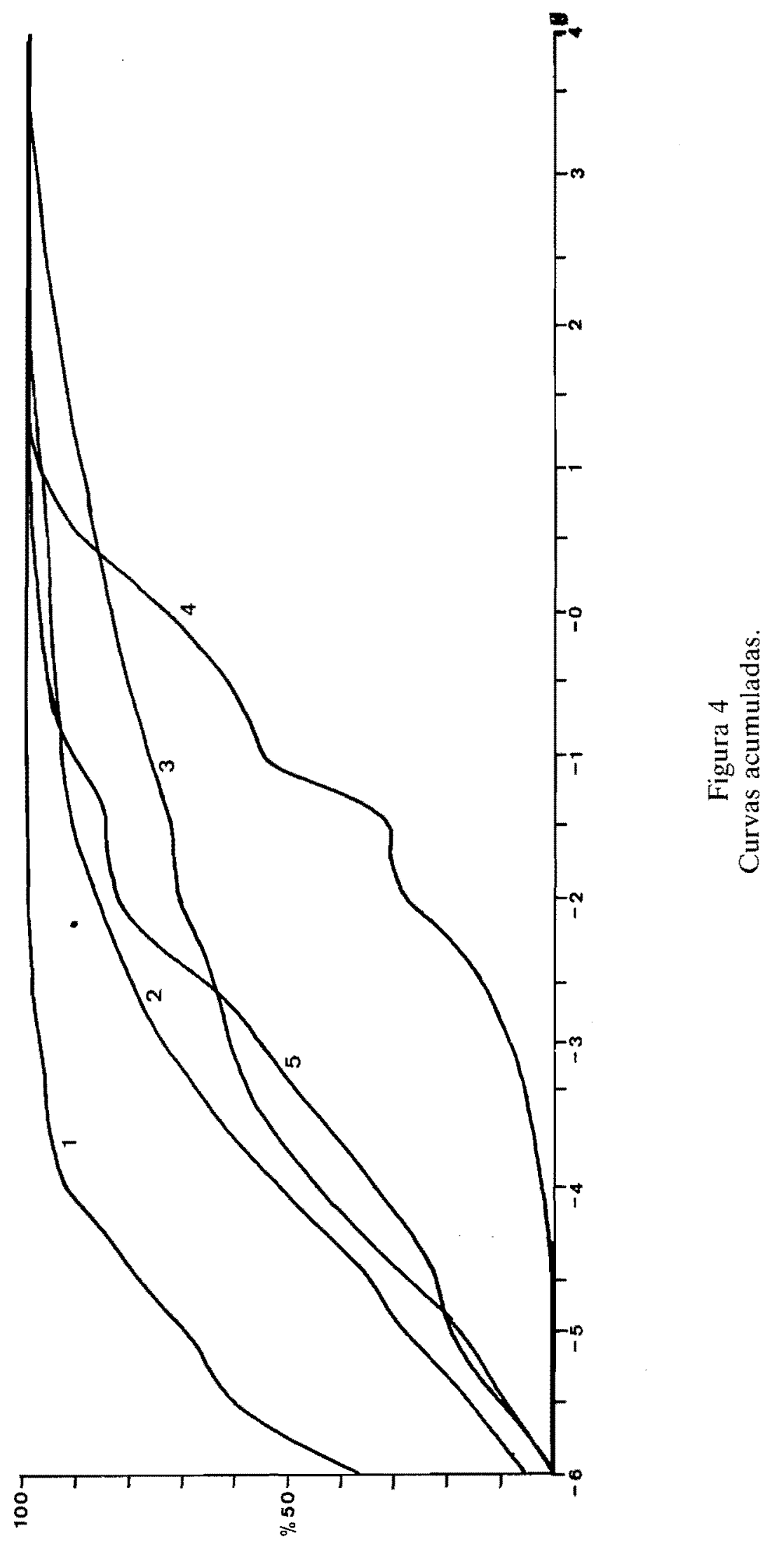


La muestra 2 se localiza en el extremo proximal de la barra. Su calibre medio es sensiblemente inferior al de la muestra precedente con $-3,93$. El histograma de frecuencia - polimodal - indica una mayor heterometría (fig. $3)$. En esta muestra hay mayor proporción de fracción arena y, en conjunto, está pobremente clasificada. La curva acumulada se dibuja tendida - como consecuencia de su escasa selección- aunque sigue manteniendo una tendencia parabólica (fig. 4). Su asimetría continúa siendo positiva, por lo que en su depósito la acumulación predominó sobre el lavado. Ninguna de estas dos muestras presenta inflexiones, por lo que es evidente que no han existido ni remociones ni mezclas con otros materiales, y que todo el depósito obedece a una única crecida que no ha sufrido retoques posteriores. La saltación ha sido la modalidad de transporte preferente.

La disposición textural de los sedimentos de esta barra -más gruesos en el extremo distal y más finos en el proximal- se ajusta perfectamente a la teoría propuesta por LEOPOLD; WOLMAN; MILLER (1964, p. 115), sobre la génesis de las barras. El material más grueso constituye un núcleo que retiene una cola de sedimentos más finos. Hay que hacer constar, sin embargo, que la presencia de árboles en el cauce puede haber favorecido la retención de los calibres más groseros, coadyuvando a la formación de la barra.

La muestra 3 se corresponde con un pequeño canal lateral ubicado en la margen derecha del cauce. Su calibre medio es de $-2,97 \varphi$. bastante semejante al del extremo proximal de la barra en cuyas inmediaciones se localiza. Esta muestra es sumamente heterométrica y polimodal (fig. 3) y su clasificación mala. La curva acumulada se presenta muy tendida y presenta algunas inflexiones (fig. 4). En este caso hay indicios de mezclas o remociones con sedimentos aportados en algún otro momento. En estos materiales el rodamiento afectó al $50 \%$ del total, con un punto de ruptura en $-3,54$, la saltación a un $34 \%$ y la suspensión a un $6 \%$, localizándose el punto de ruptura entre ambas modalidades en 0 .

La muestra 5 corresponde al canal lateral de la margen izquierda, que por su aspecto parecía ser el canal activo. La talla media es de $-3,4 \varphi$, algo superior a la del canal derecho. Se trata, asimismo, de una muestra muy heterométrica con histograma polimodal (fig. 3), pobre clasificación y con señales de remoción.

Por último, la muestra 4 corresponde a un cuerpo arenoso adosado a la barra principal, que por su aspecto y morfología, hace pensar en una formación de tipo media luna (crescentic). A excepción del calibre medio $(-1,08 \varphi)$, presenta las mismas características que la muestra anterior. En ambos casos todo el material va en saltación.

Resulta curioso observar que en este tramo de la Rambla de Chiva - al contrario de lo que ocurre aguas abajo, como puede comprobarse en las con- 
clusiones del trabajo de SANJAUME; SEGURA; MEYER (ver" en este mismo volumen) - los canales presentan un calibre medio inferior al de la barra. Este hecho, unido a la moderada energía de flujo que denota la modalidad de transporte predominante en los citados canales, hace pensar que la máxima energía de flujo se concentró en la parte central del cauce.

Es obvio que durante el pico de crecida el agua ocupaba todo el cauce, con una altura aproximada de $2 \mathrm{~m}$., como demuestran los restos de suciedad que han permanecido entre las ramas de los árboles. Sin embargo, al disminuir el caudal, dada la configuración ligeramente curvada del cauce y la existencia - aguas arriba - de una barra transversal, el flujo de dirigía hacia la margen izquierda del cauce y allí se reflejaba siguiendo a continuación una trayectoria que desde esta orilla se desviaba paulatinamente hacia la derecha, sin constreñirse a los canales. Los crescent marks que se han formado en la superficie de la barra corroboran que un flujo de bastante energía circuló sobre la misma. Por otra parte, el grueso calibre medio de los sedimentos de esta barra puede estar relacionado con la turbulencia generada por la presencia de los árboles.

\section{Conclusiones}

Como bien afirma ROSSELLÓ $(1983$, p. 6), las inundaciones no son desastres de la naturaleza, sino que son acontecimientos normales en el comportamiento de los ríos. Es la acción antrópica la que, haciendo un uso indebido del territorio, provoca las catástrofes. En la avenida que nos ocupa se pueden distinguir también estos dos aspectos.

Por lo que respecta a los factores naturales, la conjunción de varios de ellos dio lugar a la avenida. Por un lado, las fuertes y concentradas precipitaciones - más de $100 \mathrm{~mm}$. en Chiva caídos en un cuarto de hora- aportaron un caudal extraordinario al cauce. Por otro, la disposición ortogonal de la red fluvial y el trazado dificultoso del canal principal agravaron esta situación inicial.

La acción antrópica contribuyó a alterar, todavía más, e! precario equilibrio de la rambla. La construcción de una fábrica sobre el cono del Barranco de Cañadillas, la nacional III y la vía del ferrocarril son los responsables de la inundación de la población de Chiva, en la medida que desviaron las aguas hacia ella.

En las consecuencias de esta avenida también podemos distinguir claramente entre las que afectaron a la rambla —en ningún modo desastrosasy las que afectaron a la población. Para confirmar el primer supuesto se ha estudiado una sección transversal del cauce después de la crecida. La disposición textural de los sedimentos parece sugerir que la energía del flujo no fue uniforme en toda la sección, sino que fue máxima sobre una barra exis- 
tente en las proximidades de la margen derecha. Sobre ella se han formado marcas de erosión - crescent marks - a causa de los árboles que allí crecen. En ellos han quedado señales que demuestran que el agua sobrepasó los 2 $\mathrm{m}$. de altura. En el curso inferior, el agua inundó los campos circundantes en busca del Barranc de Torrent, ante la falta de un cauce bien definido en este tramo de la rambla.

Por lo que respecta a la población, las pérdidas fueron de orden material - a diferencia de anteriores avenidas en las que hubo víctimas mortales-y afectaron principalmente a la población de Chiva y a las de la cuenca baja. En ellas se produjeron daños en la agricultura, la industria, servicios y otros bienes. De todo lo expuesto anteriormente podemos concluir que el hombre, en la ocupación del territorio, debe ser sumamente respetuoso con la naturaleza y sus leyes. La transgresión de las mismas, supone siempre un grave riesgo.

\section{Bibliografía}

LEOPOLD, L.B.; WOLMAN, M.G.; MILLER, J.P. (1964): Fluvial Processes in Geomorphology, San Francisco, W.H. Freeman, 522 pp.

MATEU BELLES, J.F. (1982): El Norte del País Valenciano. Geomorfología litoral y prelitoral, Valencia, Universidad de Valencia, $286 \mathrm{pp}$.

MATEU BELLES, J.F. (1983): Aluvionamiento medieval y moderno en el llano de inundación del Júcar, Cuad. de Geogr., 32-33, pp. 291-310.

PEREZ CUEVA, A.; ARMENGOT, R. (1983): El temporal de octubre de 1982 en el marco de las lluvias torrenciales en la cuenca baja del Júcar. Cuad. de Geogr., 32-33, pp. 61-86.

ROSSELLO VERGER, V.M. (1983): La revinguda del Xúquer i el desastre de la Ribera (20-21 octubre 1982). Una perspectiva geogràfica. Cuad. de Geogr., 3233 , pp. 3-38. 